\section{Carrier detection and prenatal diagnosis of PKU by phenyalanine hydroxylase locus specific DNA polymorphism}

\section{FLEMMING GÜTTLER}

\section{The John F. Kennedy Institute, DK-2600 Glostrüp, Copenhagen, Denmark}

\section{SALVIO L.C. WOO}

\section{Howard Hughes Medical Institute, Department of Cell Biology, Baylor College of Medicine, Houston, Texas, USA}

Recent advances in the study of human genomic DNA suggest that anyone individual has a variant but inherited nucleotide pair for every 100-300 of our $3 \times 10^{9}$ nucleotide pairs. These nucleotide variations will either destroy or create recognition sites for restriction enzymes that cleave genomic DNA at precisely defined sequences of 4-6 nucleotide pairs. By analyzing genomic DNA with one restriction enzyme and a radioacctive cDNA probe, different length fragments of DNA will appear according to the presence or absence of these recognition sites. The individual restriction fragment length polymorphism (RFLP) obtained provides a source of genetic markers that can be used to trace mutant genes to which they are linked through successive generations of families.

Eight restriction enzymes have revealed polymorphic patterns at the phenylalanine hydroxylase gene using a cDNA probe, i.e. a copy of the phenylalanine hydroxylase gene (3). The mutations responsible for PKU is not detected by this type of analysis. However, since the cDNA probe contains the coding sequences of the phenylalanine hydroxylase gene, the detected polymorphisms are due to harmless nucleotide variations in the gene that segregate in a Mendelian fashion. Thus, the polymorphic patterns have been used to trace the transmission of the mutant gene in PKU-families and RFLP haplotype analyses of the phenylalanine hydroxylase gene in PKUf-families have succesfully been used for prenatal diagnosis and carrier detection of this inherited metabolic disorders ( 1 , 2,3 ).

The observation of multiple RFLPs at the phenylalanine hydroxylase gene has offered the possibility for haplotype analysis. An individual will be either homozygous or heterozygous with respect to the polymorphic fragments obtained after digestion with one restriction endonuclease. The combination of the restriction fragments obtained after digestion of an individual's genomic DNA with each of the eight restriction enzymes form a haplotype. By comparing the haplotypes associated with the mutant phenylalanine hydroxylase genes of a PKU-child with the haplotypes associated with the normal and the mutant genes of the parents, it is possible for each parent to assign the haplotype associated with the normal gene and the haplotype associated with the mutant gene ( 1 , 2).

To establish the usefulness of DNA polymorphism in diagnosis of PKU the haplotypes have been determined in 38 Danish families, each with one or more affected sibs. Parental haplotypes of 37 each with one or more affected sibs. Parental haplotypes of 37 families were completely informative, i.e. permitting determination of affected, carrier and non-carrier sibs. No evidence for recombination was found in any of these families. Theoretical1y, the observed haplotypes will establish disease status in $87 \%$ (2)

Haplotype analysis in the Danish PKU-families revealed 12 different haplotypes. However, of the 152 chromosomes analysed 118 were associated with 4 haplotypes. A majority of the PKU-genes were associated with 2 of these common haplotypes, both of which were different from the predominant haplotypes associated with the normal genes. Preliminary investigations suggest a relationship between the phenotype of the affected child and the haplotypes associated with the mutant genes inherited from the parents (1). The observations of different haplotypes associated with B-thalassemia led to the strategy of sequencing only those mutant genes present in different haplotypes. This strategy has produced a high yield of previously undiscovered mutations in B-thalassemia. If the PKU genes in the Danish population are the result of multiple mutations which occurred on chromosomes of the most common haplotypes, the same strategy is potentially applicable for the molecular characterization of the various types of phenylalanine hydroxylase. deficiency.

1. Güttler F, Lidsky AS, Woo SLC 1985 Molecular genetics of PKU: Prenatal diagnosis and carrier detection by gene analysis. I "Recent Progress in the Understanding, Recognition and Management of Inherited Diseases of Anino Acid Metabolsim" (Bickel $\mathrm{H}$, Wachtel $\mathrm{U}$ eds) Georg Thieme Verlag, Stuttgart and New York,

2. Lidsky AS, Ledley FD, DiLella AG, Kwok SC, Daiger SP, Robson $\mathrm{KJH}$, Woo SLC 1985 Extensive restriction site polymorphism at the human phenylalanine hydroxylase locus and application in the human phenylalanine hydroxylase locus and application

3. Woo SLC, Lidsky AS, Güttler F, Chandra T, Robson KJH 1983 Cloned human phenylalanine hydroxylase gene allows prenatal diagnosis and carrier detection of classical phenylketonuria. Nature 306:151

\section{X-Linked Agammaglobulinemia: Mapping of the Gene for XLA Carrierdetection}

RUUD K.B. SCHUURMAN, EWALD J.B.M. MENSINK, ALLAN THOMPSON, MARGRIET E.M. KRAAKMAN, JOHANNES D.L. SCHOT

\section{Division of Immunobiology Department of Immunohaematology, University Medical Center, Leiden and Department of Pediatrics, Sophia Childrens Hospital,} Rotterdam, the Netherlands

$X-1$ inked Agammaglobulinemia or Panhypogammaglobulinemia is a severe antibody deficiency disease associated with protracted and recurrent bacterial infections. The disease was unraveled in 1952 by showing the absence of serumgammaglobulins in a male patient. Successively it was demonstrated that patients had serumlevels of immunoglobulin isotypes less than the lower limits of the normal ranges for age, that patients lacked plasmacells in iymphoid tissues, that they lacked antibodies to various microorganisms or toxins including diphteria, whooping cough, tetanustoxoid and poliovirus against which patients had been vaccinated, that papolion in peripheral blood, but that they had IgM-heavy chain containing pre-B lymphocytes in bone marrow.

Investigations at the molecular genetic level indicate that most XLA-B lymphocytes are arrested during $B$ iymphocyte differentiation at the pre (pre) B cell stage. Pre (pre) B lymphocytes have no functional immunoglobulin variable region rearrangements and may express truncated immunoglobulin - constant region - fragments. However, the genetic defect is not absolute; small numbers of $B$ iymphocytes proceed in differentiation by variable region rearrangements of the immunoglobulin heavy and light chains. Very small numbers of surface-Ig-positive $B$ lymphocytes have been small numbers of shown to be able to secrete complete IgM molecules. Al though it shown to be able to secrete complete IgM molecules. Although it lin gene rearrangements, no clear function of the XLA gene has as yet been delineated, which precludes direct isolation and characterization of the gene.

The XLA gene segregates in families as an $X$ linked recessive trait. According to Lyon's hypothes is female XLA carriers should on the average have half of the normal numbers of mature $B$ lymphocytes and plasmacells. We have investigated the established female carriers within an extensive 8 generation pedigree with more than 34 Agammaglobulinemia males and found no immunologic abnormalities in the XLA carriers.

Thus to provide a method for XLA carrier detection markers encoded by genes segregating with the XLA gene should be searched for. Such markers should be in close linkage with the XLA gene as to make the chance for recombination between the marker and the XLA gene during meiosis as small as possible. The 8 generation pedigree provided an exceptional model for such studies in that it could be demonstrated that all patients and carriers actually it could be demonstrated that all patients and carriers actually
carried the identical defect within the same genuine $X$ linked gene. This excludes genetic complexity, a problem in linkage studies encountered when multiple pedigrees are analysed. Furthermore, pedigree analysis showed that without therapy - antibiotics on months and 8 years of age, an information now used in the reconstruction of the segregation pattern in other pedigrees. We have shown that the genetic distance, which reflects recombination frequencies, between $X g(a)$ and $12 E 7$ markers located at the telomeric site of the short arm of the $X$ chromosome and XLA is at least 20 cent $i$-Morgans (1). At present we use $X$ chromosomal derived DNA probes recognizing polymorphic restriction sites (RFLP). RFLP studies enable us to walk from the telomeric site of the short arm of the $X$ chromosome to the centromere and further along the long arm of the chromosome. The available data suggest that the XLA gene is located around the centromere. Furthermore, from an analysis of multiple pedigrees heterogeneity of XLA emerges.

1. Mensink EJBM, Schot JDL, Tippett P, Ott J, Schuurman RKB 1984 $X$ linked Agammaglobul inemia and the red blood cell determinants $\mathrm{Xg}$ and $12 \mathrm{E} 7$ are not closely linked. Human Genetics 68: 303-309. 